\title{
Constraints on a long-range spin-independent interaction from precision atomic physics
}

\author{
S. G. Karshenboin円 \\ D. I. Mendeleev Institute for Metrology, St.Petersburg, 190005, Russia \\ and Max-Planck-Institut für Quantenoptik, Garching, 85748, Germany
}

\begin{abstract}
Constraints on a spin-independent interaction by exchange of a neutral light boson are derived from precision data on the electron anomalous magnetic moment and from atomic spectroscopy of hydrogen and deuterium atoms. The mass range from $1 \mathrm{eV} / c^{2}$ to $1 \mathrm{Mev} / c^{2}$ is studied and the effective coupling constant $\alpha^{\prime}$ is allowed below the level of $10^{-11}-10^{-13}$ depending on the value of the boson mass. The mass range corresponds to the Yukawa radius from $0.0002 \mathrm{~nm}$ to $20 \mathrm{~nm}$, which covers the distances far above and far below the Bohr radius of the hydrogen atom.
\end{abstract}

PACS numbers: $12.20 .-\mathrm{m}, 31.30 . J-, 06.20 . \mathrm{Jr}$

\section{INTRODUCTION}

Precision tests of quantum electrodynamics for bound states and free particles allow us to verify various advanced methods of measurements and calculations and determine precision values of certain fundamental constants, such as the Rydberg constant $R_{\infty}$ and fine structure constant $\alpha$. The overall consistency of the results obtained for those constants [1], proves, in particular, that we can consistently describe various fundamental phenomena in a broad range of distances and energies [2].

Meanwhile, an introduction of new physics could affect various scales with different strength and violate the consistency mentioned. If such a problem is not observed, one sets various constraints on possible new physics.

In particular, various unification theories suggest additional particles (see, e.g., [3 [5]). A specific kind of such particles is a light neutral particle weakly interacting with conventional matter consisting of electrons and nucleons. Indeed, 'lightness' in terms of particle physics ranges from below $1 \mathrm{eV} / c^{2}$ to above $1 \mathrm{GeV} / c^{2}$. Stable neutral particles of this kind can be also considered as a candidate for the dark matter $[6]$.

In a broad range of distances one can consider an additional new interaction as a modification of the Coulomb interaction such as

$$
\frac{\alpha}{r} \rightarrow \frac{\alpha_{\text {eff }}(r)}{r}=\frac{\alpha}{r}+\frac{\alpha^{\prime}}{r} e^{-\lambda r} .
$$

A similar substitute can be also written in the momentum space.

The particle is not necessarily stable in our consideration. Generally, one should rather expect that a particle coupled to charged particles, decays into a few photons, while a particle coupled to leptons, could also decay to neutrinos. (Decay into a pair of massive charged leptons can be forbidden for light particles because of their lightness.) The substitute (1) is valid as long as the decay width is much smaller than the mass $\lambda$.

\footnotetext{
*Electronic address: savely.karshenboim@mpq.mpg.de
}

Comparing values of the fine structure constant $\alpha$ obtained from experiments with different characteristic distances and momenta, one can check whether the Coulomb coupling constant is really a constant. (We have in mind that the vacuum polarization corrections responsible for the 'running coupling constant' have been already taken into account.)

The most precise measurements of various physical quantities are often aiming at the determination of the values of fundamental constants. The related precision data open a certain window of opportunities to verify the constancy of the Coulomb coupling constant. There are basically three important scales, which contribute to precision QED-related experiments.

The scale of distances related to the Bohr radius $a_{0}=1 /\left(\alpha m_{e} c\right)$ is studied within hydrogen spectroscopy with transitions involving the ground and the low excited states $(1 s, 2 s)$. The distances of about $10^{3} a_{0}$ are accessible in experiments on high Rydberg states in the hydrogen atom $(n \simeq 30)$, while the Compton wave length of the electron $\lambda_{C}=\hbar /\left(m_{e} c\right)$ is a characteristic distance involved in calculations of the electron anomalous magnetic moment.

Altogether, the related distances vary from $2 \times 10^{-13} \mathrm{~m}$ to $2 \times 10^{-7} \mathrm{~m}$ and related masses range from $1 \mathrm{eV} / c^{2}$ to $1 \mathrm{MeV} / c^{2}$. Indeed, in the case of the completely relativistic calculation of a correction to the electron anomalous magnetic moment we cannot rely on a static component in (1), but should deal with an interaction describing an exchange by an intermediate particle.

Let us now consider precision QED-related experiments for these three areas step by step.

\section{ANCHOR EXPERIMENTS: MEASUREMENTS ON LOW STATES IN THE HYDROGEN ATOM (DISTANCES $(1-4) a_{0}$ )}

A massive set of data on hydrogen and deuterium spectra is available for low excited states related to physics from one to a few Bohr radii. They are used here as an anchor for further comparison with other dis- 
tance/momentum scales. We remind that the Bohr radius $a_{0} \simeq 0.53 \times 10^{-10} \mathrm{~m}$ is a characteristic distance for the electron orbit at the ground state. For the $2 s$ state the characteristic distance is $\sim 4 a_{0}$.

Throughout the paper the relativistic units are applied in which $\hbar=c=1$. In these units $a_{0}=1 / \alpha m_{e}$. For the Yukawa radius equal to the Bohr radius, the related mass of the intermediate particle $\lambda$ is $3.5 \mathrm{keV}$, while the radius of $4 a_{0}$ corresponds to $1 \mathrm{keV}$.

To understand the procedure of evaluation, one can have in mind a simple picture with the non-relativistic Schrödinger equation, because all corrections beyond such approximation, which are due to QED and relativistic effects, are well under control and can be introduced when necessary [1, 7, 8].

Spectroscopic data, which are the most important statistically, are related to the $1 s-2 s$ transition [9, 10] and to the $2 s-n s / d$ transitions for $n=8,10,12$ [1]. We remind that theory of hydrogen levels can be expressed in terms of the Rydberg constant $R_{\infty}$ and the proton charge radius [1, 7, 8]. Those constants are not known from other experiments with a sufficient accuracy and their best values are determined from the spectroscopic data under question (see, e.g., [1]). Therefore, to separate these variables and to determine $R_{\infty}$ one has to deal with at least two different transitions [1, 8, 12].

One of the utilized transitions is $1 s-2 s$ in hydrogen and deuterium, for which the distances in the interval between $a_{0}$ and $4 a_{0}$ are involved. As to the other transitions, a certain weighted average of data for all $2 s-n s / d$ transitions is used. The value of the principal quantum number $n$ for the involved excited $n s / d$ states is substantially larger than 2, and because of that the related Coulomb contribution is not larger than $1.5 \%$ and can be neglected as a good approximation.

So, the Rydberg constant [1]

$$
R_{\infty}=10973731.568527(73) \mathrm{m}^{-1}
$$

corresponds to $r \sim(1-4) a_{0}$. This value of $R_{\infty}$ can be directly compared with another value related to a different distance (see below for a comparison with a value related to $\left.r \sim 10^{3} a_{0}\right)$.

Alternatively, one can extract a value of the fine structure constant $\alpha$ from the result (2) by applying the relation

$$
R_{\infty}=\frac{\alpha^{2} m_{e} c}{2 h}
$$

For this purpose one should combine a value of $R_{\infty}$ with $h / M$ for a certain atom/particle and with a result or a chain of results, which allows one to determine $M / m_{e}$ (see 1] for detail).

The key point in such a determination of $\alpha$ is a measurement of $h / M$. There are two high-precision independent experiments on that. The first measurement is performed on caesium atoms [13] and the most recent result leads to 13

$$
\alpha_{\mathrm{Cs}}^{-1}=137.0360000(11) .
$$

All auxiliary data are taken from [1].

The other experiment has been performed on rubidium. The most recent result is [14]

$$
\alpha_{\mathrm{Rb}}^{-1}=137.03599945(62) \text {. }
$$

The weighted average of these two results ${ }^{1}$

$$
\alpha_{\text {atom }}^{-1}=137.03599959(53)
$$

is a value of the fine structure constant related to distances from $a_{0}$ to $4 a_{0}$.

\section{PRECISION MEASUREMENTS OF HIGHLY EXCITED STATES IN THE HYDROGEN ATOM (DISTANCES $\sim 10^{3} a_{0}$ )}

There is one more result on determination of the Rydberg constant, which is usually not included into the adjustment [1] because it is somewhat less accurate than the quoted above data and, what is more important, the result is only a preliminary one. The value

$$
R_{\infty}=10973731.56834(69) \mathrm{m}^{-1}
$$

is obtained [16] from a transition between the Rydberg states with $n \simeq 30$ in the hydrogen atom. Since the value resulted from a partial evaluation of possible systematic effects, to be on the safe side the uncertainty in (7) is tripled against its original value. As it was confirmed by the authors of the experiment [17], that is rather an overconservative estimation of the uncertainty.

Let us now constrain an effective interaction with mass $\lambda$, which is

$$
4 \mathrm{eV} \ll \lambda \ll 1 \mathrm{keV}
$$

A contribution of an additional boson in such a case reads

$$
R_{\infty}= \begin{cases}\frac{\left(\alpha+\alpha^{\prime}\right)^{2} m_{e}}{2}, & \text { at } r \sim a_{0}, \\ \frac{\alpha^{2} m_{e}}{2}, & \text { at } r \sim 10^{3} a_{0} .\end{cases}
$$

That leads to a constraint [18]

$$
\alpha^{\prime}=(0.6 \pm 2.3) \times 10^{-13}
$$

That is the strongest constraint on a long-range spinindependent interaction, which can be derived from atomic physics and fundamental constants.

\footnotetext{
${ }^{1}$ A somewhat less accurate previous rubidium result $\alpha_{\mathrm{Rb}}^{-1}=$ 137.035 99883(91) from the same group [15] has not been included into averaging because of possible correlations. Once we treat that result as completely independent, the average value is $\alpha_{\text {atom }}^{-1}=137.03599939$ (46) (cf. [6) ), which does not change the final conclusions too much.
} 


\section{PRECISION PHYSICS AT THE COMPTON WAVE LENGTH (MOMENTUM $\sim m_{e} c$ )}

One can also compare physics at the Bohr radius and at the Compton wave length of an electron. A suitable tool for that is an examination of various results on the fine structure constant. We have already discussed a value related to $a_{0}$ (see (6) ). Meanwhile, the most accurate value of the fine structure constant comes from the anomalous magnetic moment of an electron

$$
\alpha_{g-2}^{-1}=137.035999084(51),
$$

obtained by combining the experimental result [19] with theory [20].

Comparing it with the value in (6) one can find a constraint for

$$
4 \mathrm{keV} \ll \lambda \ll 0.5 \mathrm{MeV} .
$$

The results of the measurements can be presented as

$$
\alpha= \begin{cases}\alpha+\alpha^{\prime}, & \text { at } q \sim m_{e}, \\ \alpha, & \text { at } q \sim 1 / a_{0},\end{cases}
$$

where we refer to momentum space and assume that the intermediate particle is a pure vector. The constraint reads [18]

$$
\alpha^{\prime}=(2.7 \pm 2.9) \times 10^{-11} .
$$

If the particle has another spin or it is a pseudovector or a combination of vector and pseudovector (such as the $Z$ boson), then the constraint is of the same order of magnitude but a factor compatible with unity can appear.

\section{FINAL CONSTRAINTS}

Our results are summarized in Table I. We can extend our analysis beyond the asymptotic limits. In particular, we also present in Fig. 1 1 results, which cover an intermediate area of a few $\mathrm{keV}$ 18.

\begin{tabular}{ccc}
\hline Range & $\alpha(r)-\alpha\left(a_{0}\right)$ & Effect \\
\hline $4 \mathrm{eV} \ll \lambda \ll 1 \mathrm{keV}$ & $(-0.6 \pm 2.3) \times 10^{-13}$ & $\mathrm{H}, n=30$ \\
$4 \mathrm{keV} \ll \lambda \ll 0.5 \mathrm{MeV}$ & $(2.7 \pm 2.9) \times 10^{-11}$ & $g_{e}-2$ \\
\hline
\end{tabular}

TABLE I: The constraint on the deviation of the effective long-range interaction $\alpha(r) / r$ from the Coulomb exchange [18]. Here, $\alpha(r)=\alpha(\infty)+\alpha^{\prime} \exp (-\lambda r)$. The $a_{0}$ scale is related to hydrogen spectroscopy for transitions involving low states $(1 s, 2 s)$. The related distance range is $r=0.5 \times 10^{-7} \mathrm{~m}$ (for $\lambda=4 \mathrm{eV}), 0.5 \times 10^{-10} \mathrm{~m}($ for $\lambda=4 \mathrm{keV})$ and $0.4 \times 10^{-12} \mathrm{~m}$ (for $\lambda=0.5 \mathrm{MeV}$ ).

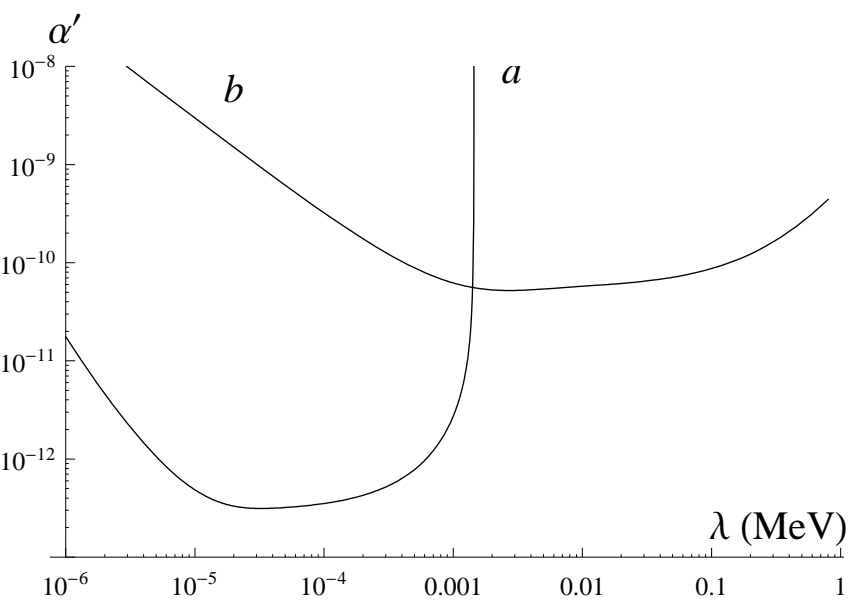

FIG. 1: Constraints on a long-term spin-independent interaction from hydrogen spectroscopy and $g_{e}-2$, including a constraint from hydrogen spectroscopy with low and Rydberg states $(a)$, a comparison of low states and $g_{e}-2(b)$. The lines are for $\left|\alpha^{\prime}\right|$ and the confidence level corresponds to one standard deviation.

To extend the constraint to a region $\lambda \sim 1 / a_{0}$ and $\lambda \sim m_{e}$, we have to modify observational equations (9) and (13).

Instead of (9) we find

$$
R_{\infty}= \begin{cases}\frac{\left(\alpha+\alpha^{\prime} \mathcal{F}_{12}\left(\lambda /\left(\alpha m_{e}\right)\right)\right)^{2} m_{e}}{2}, & \text { at } r \sim a_{0}, \\ \frac{\left(\alpha+\alpha^{\prime} \mathcal{F}_{30}\left(\lambda /\left(\alpha m_{e}\right)\right)\right)^{2} m_{e}}{2}, & \text { at } r \sim 10^{3} a_{0},\end{cases}
$$

where

$$
\begin{aligned}
\mathcal{F}_{12}(x)= & 4\left[\left(\frac{1}{1+x}\right)^{2}-2\left(\frac{1}{1+x}\right)^{3}+\frac{3}{2}\left(\frac{1}{1+x}\right)^{4}\right] \\
& -\left(\frac{2}{2+x}\right)^{2}
\end{aligned}
$$

and for $\mathcal{F}_{30}\left(\lambda /\left(\alpha m_{e}\right)\right)$ we have to consider a function

$$
\mathcal{F}_{30}(x)=\frac{\frac{1}{(n+1)^{2}}\left(\frac{2}{2+(n+1) x}\right)^{2 n+1}-\frac{1}{n^{2}}\left(\frac{2}{2+n x}\right)^{2 n-1}}{1 /(n+1)^{2}-1 / n^{2}},
$$

related to $n \simeq 30$. The transitions studied in [16] were transitions between the circular states $(l=n-1): 27 \rightarrow$ 28 and $29 \rightarrow 30$.

The related constraint is

$$
\alpha^{\prime}=\frac{(0.6 \pm 2.3) \times 10^{-13}}{\mathcal{F}_{12}\left(\lambda /\left(\alpha m_{e}\right)\right)-\mathcal{F}_{30}\left(\lambda /\left(\alpha m_{e}\right)\right)} .
$$

Eq. (13) is to be substituted for

$$
\alpha= \begin{cases}\alpha+\alpha^{\prime} \mathcal{F}_{g-2}\left(\lambda / m_{e}\right), & \text { at } q \sim m_{e}, \\ \alpha+\alpha^{\prime} \mathcal{F}_{12}\left(\lambda /\left(\alpha m_{e}\right)\right), & \text { at } q \sim 1 / a_{0},\end{cases}
$$


where the function [21]

$$
\begin{aligned}
\mathcal{F}_{g-2}(x)= & 2\left[-\left(x^{5}-4 x^{3}+2 x\right) \frac{\tan ^{-1} \sqrt{\frac{4-x^{2}}{x^{2}}}}{\sqrt{4-x^{2}}}\right. \\
& \left.+\left(x^{4}-2 x^{2}\right) \ln x-x^{2}+\frac{1}{2}\right]
\end{aligned}
$$

is the function which is applied when one calculates the hadronic vacuum polarization through integration over a dispersion variable $\lambda=\sqrt{s}$.

The related constraint is

$$
\alpha^{\prime}=\frac{(2.7 \pm 2.9) \times 10^{-11}}{\mathcal{F}_{g-2}\left(\lambda / m_{e}\right)-\mathcal{F}_{12}\left(\lambda /\left(\alpha m_{e}\right)\right)} .
$$

We summarize behavior of the profile functions $\mathcal{F}$ in Fig. 2. Each of them is equal to unity for $\lambda \rightarrow 0$ and equal to zero for $\lambda \rightarrow \infty$. The unity region is the one, where the Yukawa potential is indistinguishable from the Coulomb potential, and the effective Coulomb coupling constant there is $\alpha+\alpha^{\prime}$. In the zero region the Yukawa potential shrinks to a zero-radius potential and does not affect any measurable values.



FIG. 2: The profile functions, which determine coverage of the constraints on a long-term spin-independent interaction from hydrogen spectroscopy and the anomalous magnetic moment of an electron. The profile function $\mathcal{F}_{30}(x)$ is with $n=27$ and $n=29$.

What is different in the profile functions is the characteristic value of $\lambda$, which separates the unity region from the zero one and the shape of the transition area around it.

We present in Fig. 2 four rather than three profile functions. Function $\mathcal{F}_{30}$ is presented in two versions. That is due to the fact that the result (7) is a preliminary one and the evaluation has not yet been completed. The data included two transitions, namely $27 \rightarrow 28$ and $29 \rightarrow 30$ and it is in part uncertain what their relative weights should be in averaging. To clarify the issue, we present in Fig. 2 two versions of the profile functions $\mathcal{F}_{30}$, namely for $n=27$ and $n=29$. One sees that the results are nearly identical in the logarithmic scale. Due to that, for the final constraint in Fig. 11 we have applied a profile with $n=28$.

\section{SUMMARY}

The interaction with an intermediate particle is not necessarily universal. The hydrogen and deuterium data allow one to conclude about $\alpha_{e p}$ and $\alpha_{e d}$, while the anomalous magnetic moment deals with $\alpha_{e e}$. (The uncertainty of the anchor data involving hydrogen and deuterium is small and the accuracy of the constraint is determined in both cases above by the other pieces of data. That allows to reduce the $a_{0}$ related data to pure hydrogen data.) A possible discrepancy between these two values could be interpreted not only as related to the certain distance scale, but also to non-universality of one-particle exchange within a broader range.

We remind that the neutrality of a hydrogen atom or a neutron (see, e.g., [22]) is a property related to a macroscopic distance and, in principle, a direct test whether there is an ultraweak long-range component of interaction of a hydrogen atom or a neutron at microscopic distances is required. We will address this problem elsewhere.

For the $b$ constraint in Fig. 1, we have utilized two values of the fine structure constant, namely those from $g_{e}-2$ and from atomic physics. In principle, one can use other less accurate values (see Fig. 3] and [1] for a review of data and references) to compare with $\alpha_{g-2}$. That produces a constraint similar to the $b$ line in Fig. 1. It is weaker for $\lambda \gg a_{0}^{-1}$, but has the same profile behavior, while for $\lambda \leq a_{0}^{-1}$ the limitation increases slower than the $b$ line. However, as we see in Fig. 1] in this area the $a$ constraint is already applicable and such extension of the $b$ constraints to the lower mass range cannot improve the whole constraint.

Another issue is model dependence. The substitute (1) is to constrain a situation with a single light intermediate particle. If there are a few of them and their coupling constants are comparable, the situation becomes more complicated. Still, if the related coupling constants are of the same sign, the results of the paper are still applicable. However, if the Yukawa terms involve interactions with different radii, while the coupling constants have compatible absolute values and opposite signs, the constraint derived is completely out of validity. As an example, one can consider a long-range correction of the form

$$
\alpha^{\prime} \frac{e^{-\lambda_{1} r}-e^{-\lambda_{1} r}}{r}
$$

where the masses are of the same order (e.g., $\lambda_{2}=2 \lambda_{1}$ ). Indeed, in this case the sensitivity of atomic energy levels to $\alpha^{\prime}$ is substantially reduced in most of the area, where the constraint in Fig. 1 is effective.

Concluding we emphasize that atomic physics allows one to access distances much larger than the Bohr radius and our limitation on $\alpha^{\prime}$ is valid for distances up to $0.02 \mu \mathrm{m}$. That can be compared with the limitation on the fifth force from Casimir-effect experiments [24], which also reached the range below $1 \mu \mathrm{m}$. Comparing 


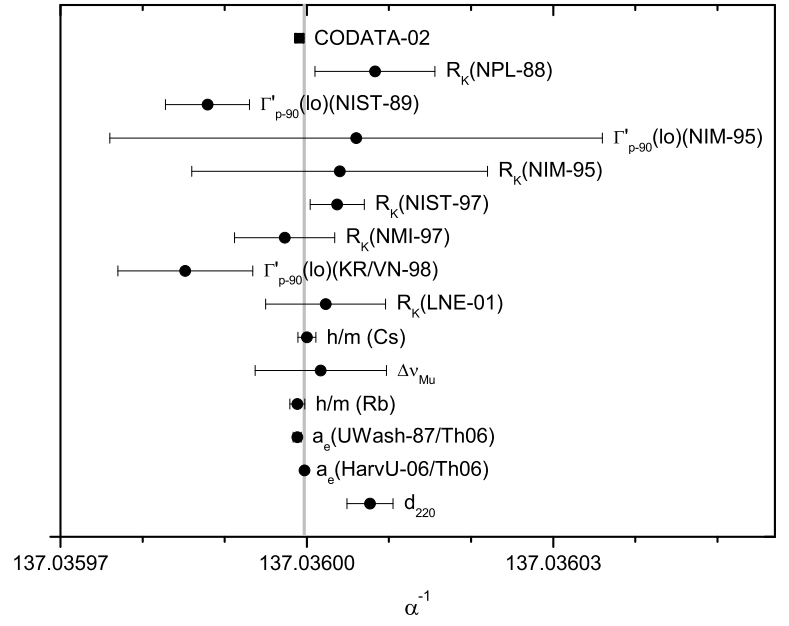

FIG. 3: Determination of the fine structure constant $\alpha$ in the CODATA-2006 adjustment 1], where the references can be found. The vertical band stands for the adjusted CODATA2006 value. The figure is reproduced from [23].

the strength, we note that in the case of Casimir effect the conventional parameterization for the Yukawa potential is $G m_{1} m_{2} \tilde{\alpha} e^{-r / \tilde{\lambda}} / r$, where $m_{i}$ is a mass of a bulk body and $G$ is Newtonian gravitation constant. There is no direct model-independent correspondence between mass-related and charge-related parameterization. The most important part of the conversion factor is $G m_{p}^{2} \sim 6 \times 10^{-39}$ (since in bulk matter there is roughly $0.5-1$ charge, e.g., baryon or lepton charge, per nucleon-the rough factor of 0.5 comes from the fact that we have an electron per from 2 to 2.5 nucleons). Using it, we find that our constraints starting from a distance below $30 \mathrm{~nm}$, where the Casimir-effect constraints stop, are weaker by a few orders of magnitude. However, they are extended to shorter distances, not accessible for the Casimir-force related experiments.

What is more important, our constraint is complementary to the Casimir-effect limitation. The latter deals with the neutral bulk matter only and cannot constrain a massive ultraweak photon $\gamma^{*}$. In contrast to that, our constraint in Table I and in Fig. 1 is covering such a case. Our results for the coupling constant $\alpha^{\prime}$ related to $\gamma^{*}$ are consistent with zero and the limitation at atomic distances is in the range of a few parts in $10^{13}$ to a few parts in $10^{11}$ depending on details as discussed above.

The keV mass range has been partly explored by means of astrophysics and cosmology. Such constraints [6, 25] mostly deal with real particles and their propagation through the matter. Details, such as the lifetime, various couplings and collision rates, are involved and they may be related to results in terms of $\alpha^{\prime}$ and $\lambda$ only through certain model-dependent relationships. Our constraints, free of such model-dependent suggestions, are complementary to those.

Another option, also not covered by Casimir effect is due to a spin-dependent long-range interaction to be considered elsewhere.

\section{Acknowledgements}

This work was supported in part by RFBR (grants \#\# 08-02-91969 \& 08-02-13516) and DFG (grant GZ 436 RUS 113/769/0-3). The author is grateful to Dan Kleppner, Andrej Afanasev, Vladimir Korobov, Masaki Hori, Astrid Lambrecht, Dmitry Toporkov, Eugene Korzinin, Simon Eidelman, and Maxim Pospelov for useful and stimulating discussions.
[1] P. J. Mohr, B. N. Taylor, and D. B. Newell, Rev. Mod. Phys. 80, 633 (2008).

[2] S. G. Karshenboim, in Precision physics of simple atoms and molecules. Ed. by S. G. Karshenboim (Springer, Berlin, Heidelberg, 2007), 35-53.

[3] S. Weinberg, Phys. Rev. Lett. 37, 657 (1976).

[4] B. Holdom, Phys. Lett. 166B, 196 (1986).

[5] M. Pospelov, Phys. Rev. D80, 095002 (2009).

[6] M. Pospelov, A. Ritz, and M. Voloshin, Phys. Rev. D78, 115012 (2008).

[7] M.I. Eides, H. Grotch, and V.A. Shelyuto, Phys. Rep. 342, 63 (2001);

M.I. Eides, H. Grotch, and V.A. Shelyuto, Theory of Light Hydrogenic Bound States, Springer Tracts Mod. Phys. 222 (Springer, Berlin, Heidelberg, 2007).

[8] S. G. Karshenboim, Phys. Rep. 422, 1 (2005).

[9] M. Niering, R. Holzwarth, J. Reichert, P. Pokasov, Th. Udem, M. Weitz, T. W. Hänsch, P. Lemonde, G.
Santarelli, M. Abgrall, P. Laurent, C. Salomon, and A. Clairon, Phys. Rev. Lett. 84, 5496 (2000);

M. Fischer, N. Kolachevsky, M. Zimmermann, R. Holzwarth, T. Udem, T. W. Hänsch, M. Abgrall, J. Grünert, I. Maksimovic, S. Bize, H. Marion, F. P. Dos Santos, P. Lemonde, G. Santarelli, P. Laurent, A. Clairon, C. Salomon, M. Haas, U. D. Jentschura, and C. H. Keitel, Phys. Rev. Lett. 92, 230802 (2004).

[10] A. Huber, T. Udem, B. Gross, J. Reichert, M. Kourogi, K. Pachucki, M. Weitz, and T. W. Hänsch, Phys. Rev. Lett. 80, 468 (1998).

[11] R. de Beauvoir, F. Nez, B. Cagnac, F. Biraben, D. Touahri, L. Hilico, O. Acef, A. Clairon and J. J. Zondy, Phys. Rev. Lett. 78, 440 (1997);

C. Schwob, L. Jozefowski, B. de Beauvoir, L. Hilico, F. Nez, L. Julien and F. Biraben, Phys. Rev. Lett. 82, 4960 (1999); 86, 4193 (2001) (Errata).

[12] S. G. Karshenboim, JETP 79, 230 (1994); Z. Phys. D39, 
109 (1997).

[13] A. Wicht, J. M. Hensley, E. Sarajlic, and S. Chu, Phys. Scr. T102, 82 (2002);

A. Wicht, E. Sarajlic, J. M. Hensley, and S. Chu, Phys. Rev. A72, 023602 (2005).

[14] M. Cadoret, E. de Mirandes, P. Cladé, S. GuellatiKhélifa, C. Schwob, F. Nez, L. Julien, and F. Biraben, Phys. Rev. Lett. 101, 230801 (2008).

[15] P. Cladé, E. de Mirandes, M. Cadoret, S. GuellatiKhélifa, C. Schwob, F. Nez, L. Julien, and F. Biraben, Phys. Rev. Lett. 96, 033001, (2006).

[16] J. Ch. De Vries, A precision Millimeter-Wave Measurement of Rydberg Frequency. Ph. D. Thesis, MIT, 2001; available at http://dspace.mit.edu/handle/1721.1/4108.

[17] D. Kleppner, private communication.

[18] S.G. Karshenboim, Phys. Rev. Lett. (2010) to be pub- lished; eprint arXiv:1005.4859.

[19] D. Hanneke, S. Fogwell, and G. Gabrielse, Phys. Rev. Lett. 100, 120801 (2008).

[20] T. Aoyama, M. Hayakawa, T. Kinoshita, and M. Nio, Phys. Rev. Lett. 99, 110406 (2007).

[21] C. Bouchiat and L. Michel, J. Phys. Rad. 22, 121 (1961).

[22] C. Amsler et al. (Particle Data Group), Phys. Lett. B667, 1 (2008).

[23] S. G. Karshenboim, Eur. Phys. J. Spec. Top. 172, 385 (2009).

[24] R. S. Decca, D. López, E. Fischbach, G. L. Klimchitskaya, D. E. Krause, and V. M. Mostepanenko, Phys. Rev. D 75, 077101 (2007).

[25] G.G. Raffelt, Ann. Rev. Nucl. Part. Sci. 49, 163 (1999). 